\title{
Processing of Surface EMG Through Pattern Recognition Techniques Aimed at Classifying Shoulder Joint Movements
}

\author{
Diletta Rivela, Alessia Scannella, Esteban E. Pavan, Member, IEEE, \\ Carlo A. Frigo, Paolo Belluco, Giuseppina Gini, Member, IEEE
}

\begin{abstract}
Artificial arms for shoulder disarticulation need a high number of degrees of freedom to be controlled. In order to control a prosthetic shoulder joint, an intention detection system based on surface electromyography (sEMG) pattern recognition methods was proposed and experimentally investigated. Signals from eight trunk muscles that are generally preserved after shoulder disarticulation were recorded from a group of eight normal subjects in nine shoulder positions. After data segmentation, four different features were extracted (sample entropy, cepstral coefficients of the 4th order, root mean square and waveform length) and classified by means of linear discriminant analysis. The classification accuracy was $92.1 \%$ and this performance reached $97.9 \%$ after reducing the positions considered to five classes. To reduce the computational cost, the two channels with the least discriminating information were neglected yielding to a classification accuracy diminished by just $4.08 \%$.
\end{abstract}

\section{INTRODUCTION}

The upper limb loss, or its congenital absence, represents a remarkable impairment for an individual, as it prevents from carrying out a wide variety of activities of daily life. Nowadays, upper limb prostheses result to be the most effective solutions to this problem, but the higher the level of the damage, the more crucial is the need of an active prosthesis. Nevertheless, as far as amputation at shoulder level is concerned, little effort has been dedicated to the control system. This may be due to the relatively low incidence of major upper limb amputation and to the difficulty to control a high number of degrees of freedom.

In order to increase user acceptance, less demanding electromyographic control methods, able to detect the patients' intention should be devised. This could be done by recognizing patterns from SEMG of synergistic muscles spontaneously activated in correspondence with a predefined motor task, for example, achieving a position in space.

In the case of major upper limb amputations, trunk muscles are particularly suitable to this purpose, as they are usually preserved, and their activities are tightly related to the intentional movements of the shoulder. A control

D. Rivela, A. Scannella are with the Dipartimento di Elettronica, Informazione e Bioingegneria, Politecnico di Milano, I-20133, Milan, Italy (e-mail: diletta.rivela@mail.polimi.it; alessia.scannella@mail.polimi.it ).

C. A. Frigo, E. E. Pavan, are with the Movement Biomechanics and Motor Control Laboratory, Politecnico di Milano, I-20133, Milan, Italy (email: carlo.frigo@polimi.it; pavan@biomed.polimi.it ).

P. Belluco is with the B10nix, I-20131, Milan, Italy (e-mail: p.belluco@b10nix.com ).

G. Gini is with the Artificial Intelligence and Robotics Laboratory Politecnico di Milano, I-20133, Milan, Italy (e-mail: gini@elet.polimi.it). strategy could take advantage of this information in order to control the prosthetic limb position [2], [3].

Aim of this study was to experimentally verify whether a pattern recognition approach could be useful to classify a number of different shoulder movements. To this purpose we have implemented a classification algorithm and then checked the possibility to reduce the number of muscles to be analyzed without losing classification accuracy.

\section{BACKGROUND}

\section{A. Pattern Recognition}

The myoelectric prostheses control underwent a significant improvement after the introduction of SEMG pattern recognition strategies [1], [4], [7]. This approach is based on the assumption that patterns of SEMG signals from several muscles include much information about the intentional movement of the prosthesis. The aim of this method is to match each SEMG pattern to one motion class among a multiplicity of preselected movements. Then the chosen movement is performed automatically in a preprogrammed mode by the prosthetic device.

Unlike the conventional myoelectric control, this method exploits the contraction of several synergistic muscles related to the movement the amputee wishes to perform, and does not require that a single degree of freedom is independently controlled. In this way, a more intuitive and rapid control can be obtained. Generally, a pattern recognition-based control approach involves these subsequent stages:

1) Multi-channel SEMG acquisition of several upper limb movements. To improve the classification accuracy, the higher the number of motion classes to discriminate, the greater the number of SEMG acquisition channel that must be recorded.

2) Data segmentation. Each channel has to be segmented into a series of time windows, adjacent or overlapping, from which a classification will be made. The window length has to satisfy real-time constraints that require the delay between command and actuation not to be greater than $300 \mathrm{~ms}$, otherwise a movement delay will be perceived by the user [8]. To use a window length greater than $250 \mathrm{~ms}$, windows must be overlapped. Smith et al. [6] suggested that the optimum window length for pattern recognition control is between $150 \mathrm{~ms}$ and $250 \mathrm{~ms}$, depending on the skill of the subject. Phinyomark et al. [7] compared eight different combinations of window lengths and increments, and 
highlighted that the best result in terms of robustness is obtained with a length of $500 \mathrm{~ms}$ and an increment of 125 $\mathrm{ms}$. The processing of myoelectric signal can be done including transients (i.e. at the contraction onset) [2] or steady states (i.e. during maintained contraction). Englehart et al. [5] showed that steady state data are classified more accurately than transient data, and, in the last case, classification suffers from less degradation with shorter data segments.

3) Feature representation. This phase involves two stages: feature extraction and feature reduction. The first stage is used to extract the hidden information from the SEMG signal and to remove the unwanted components. Generally, features belong to three main domains: time domain, frequency domain and time-frequency domain. The time domain (TD) features, based on the signal amplitude, have been widely used in most previous studies due to their relative computational simplicity [4], [9], [10].The second stage is usually implemented to reduce the dimensionality of the initial feature space, attempting to preserve classification accuracy, while reducing the computational costs and the complexity of the classifier [11]-[13].

4) Classification. The classifier receives as input the reduced feature set and it has to match the different patterns with the correct movement class as much accurately as possible.

\section{METHODS}

\section{A. Subjects and Data Acquisition}

The experimental data were collected at the Movement Biomechanics and Motor Control Laboratory of Politecnico di Milano (Milan, Italy). Eight healthy subjects (four males and four females) aged 25.0 \pm 1.8 yrs took part in this experiment. They were informed about the experimental procedures and agreed to sign a written consent. All subjects declared to have had neither neuromuscular deficit nor shoulder trauma.

Subjects performed a series of eight shoulder movements that were repeated ten times each. Every movement included four sequential phases, namely: resting, elevation, isometric holding and, finally, return to the rest condition. A set of eight movements were selected for analysis among the upper limb movements performed in the daily-life which mainly involve the shoulder joint: shoulder flexion (forward elevation, in the sagittal plane) by $45^{\circ}, 90^{\circ}$, and $110^{\circ}$; shoulder hyperextension (backward elevation, in the sagittal plane) by $-30^{\circ}$; shoulder abduction (elevation in the frontal plane) by $45^{\circ}$ and $90^{\circ}$; shoulder elevation in a $45^{\circ}$ externally rotated plane, by $45^{\circ}$ and $90^{\circ}$. We focused on these movements because, according to [14], usual prosthetic solutions for shoulder disarticulation can reasonably control only 2 degrees of freedom. For this reason internal/external humeral rotation was not considered. Subjects were instructed to perform the movements with the hand relaxed, to exclude the activation of forearm muscles; in that way, the hand was always in the neutral position, i.e. naturally pronated without actively rotating the forearm, with the palm of the hand always perpendicular to the plane of elevation. No humeral axial rotation occurred in these conditions.

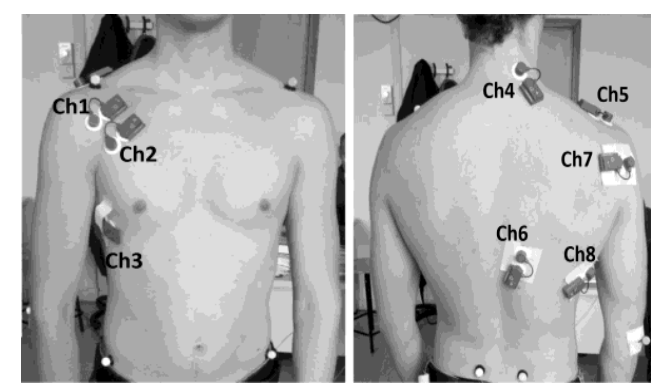

Fig. 1. Placement of the SEMG electrodes.

After cleaning the skin surface, eight pairs of electrodes were placed over the following trunk muscles (see Fig. 1): clavicular and sternal heads of pectoralis major (Ch 1 and $\mathrm{Ch}$ 2 respectively), serratus anterior ( $\mathrm{Ch} 3$ ), trapezius descendent (Ch 4), trapezius transversalis (Ch 5), trapezius ascendent (Ch 6), infraspinatus (Ch 7), and latissimus dorsi (Ch 8). These muscles are indeed synergistic in the analyzed movements and they are superficial muscles preserved also after upper limb amputation [15].

SEMG data were recorded by bipolar disposable pregelled $\mathrm{Ag} / \mathrm{AgCl}$ surface electrodes (26 mm diameter, Bielsan, Italy). They were connected to miniaturized, wireless probes (BTS- FREEEMG 300, Italy) for digital data collection. Each pair of electrodes was placed according to the guidelines provided by the Atlas of muscle innervation zones [16] with an interelectrode distance of $26 \mathrm{~mm}$.

Raw SEMG signals were recorded at a sampling rate of $1.0 \mathrm{kHz}$ and stored for off-line data processing in Matlab (The MathWorks, Natwick, USA).

\section{B. Data Segmentation}

Data analysis considered the only steady state phase (isometric hold) in order to be consistent with other studies described in literature (see [1], [5]). This phase lasted about $3 \mathrm{~s}$.

Data segmentation was performed selecting a window length $\mathrm{L}=500 \mathrm{~ms}$ and an increment $\mathrm{I}=62 \mathrm{~ms}$.

After a random shuffling of all acquired trials, data were split into a training dataset, the first $60 \%$, and a test dataset, the last $40 \%$. In order to verify whether the choice of training data could affect our results, a cross-validation was performed using different portions of data for training and testing. Ten independent classifications were performed using a $60 \%$ training window shifted of $10 \%$ of all data. After feature extraction from all datasets, the training set was used to reduce the dimensionality of the original feature set and to train the classifier. Instead, the test dataset was used to estimate the classification accuracy.

\section{Feature Selection}

For each of the six segmentations tested, a feature set was extracted: the TD feature set proposed in [7] for its robustness in time, i.e. sample entropy (SampEn), cepstral coefficients (CC) of the 4th order, root mean square (RMS), and waveform length (WL).

\section{Classification}

Classification was performed using linear discriminant analysis (LDA) [17]. This classifier was chosen due to its advantages: high performance in EMG signal classification, 
low computational cost, ease of implementation, high speed training and robustness [2], [7], [10]. Furthermore, it does not require iterative training, avoiding the potential for under- or over-training, and it does not need any parameter adjustment.

A study carried out by Hargrove et al. [14] compared LDA classifier with other more complex classifiers, demonstrating that there are no significant differences, in terms of classification accuracy. The authors concluded that, with an appropriate feature representation, the classification task could be reduced to a simple linear problem.

The performance of the classification system was defined in terms of classification accuracy and was evaluated by considering three different sets of motion classes. The first set was composed by all motion classes described above with the rest class in addition. The second set consisted of five motion classes: shoulder flexion at $90^{\circ}$, shoulder hyperextension at $-30^{\circ}$, shoulder abduction at $90^{\circ}$, shoulder elevation at $90^{\circ}$ along the plane rotated by $45^{\circ}$ in relation to the sagittal plane, and rest. The last set was composed by four motion classes: shoulder flexion at $90^{\circ}$, shoulder hyperextension at $-30^{\circ}$, shoulder abduction at $90^{\circ}$, and resting condition.

\section{E. Dimensionality Reduction}

After having identified the most accurate method, we have investigated the effect of SEMG channel reduction on the classification performance. To this purpose, the mean value of each feature was calculated for each motion class and each acquisition channel. Then, in order to select the most relevant channels, the standard deviation of the features was used as a metric of dispersion and the channels showing the lowest dispersion among the classes were neglected.

\section{RESULTS}

\section{A. Feature Selection}

The cross-validation showed that the differences in the classification accuracy obtained from the different training sets were less than $4 \%$, and these differences were mainly affecting the performance of the worst cases. The results shown are so related to just one of the ten different training sets: the one that considers the $60 \%$ training window at the beginning of the data.

\section{B. Classification accuracy}

The classification performance was assessed as the percentage of correctly classified motions over the tested motions included in each motion-classes subset considered.

The classification accuracy was $100 \%, 97.94 \%$ and $92.11 \%$ for four-, five- and nine- motion classes, respectively.

As expected, a deterioration in the classification accuracy was observed when increasing the number of classes.

For the five-motion, resting and hyperextension at $-30^{\circ}$ achieved an accuracy of $100 \%$. Instead, the shoulder elevation of $90^{\circ}$ in an elevation plane externally rotated by $45^{\circ}$, with a classification accuracy of $99.7 \%$, was mostly misclassified as abduction at $90^{\circ}(6.8 \%)$ or flexion at $90^{\circ}$ $(3.4 \%)$.

Finally, for the nine-motion, the most misclassified motion, with a classification accuracy of only $73.9 \%$, was the flexion at $110^{\circ}$, which was misclassified as flexion at $90^{\circ}(10.2 \%)$ or as shoulder elevation at $90^{\circ}$ in an elevation plane externally rotated $(3.1 \%)$. The classes that showed the best estimation accuracy were, respectively, resting and hyperextension at $30^{\circ}$, where the best classification accuracy was obtained $(100 \%)$.

\section{Dimensionality Reduction}

The features extracted from the SEMG signals of the selected muscles showed a different level of sensitivity to the motion classes. For each feature, the channels that showed the lowest sensitivity to the variations observed among all motion classes were detected. As dispersion metric, the standard deviation of the mean values of each feature, among all motion classes and for all subjects, were considered. The channels that showed the lowest dispersion (defined as a percentage of the standard deviation reported in the first column) were the latissimus dorsi (Ch 8), the sternal head of the pectoralis major (Ch 2) and, to a lesser extent, the trapezius transversalis ( $\mathrm{Ch} 5)$.

Thus, these channels have been removed and the processing has been reapplied. Comparing the six-channel classification system with the eight-channel one, it can be observed that: for the four-class problem, the accuracy keeps the value of $100 \%$; for the five-class problem, the channel reduction leads to a decline of classification accuracy of $2.41 \%$; while, for the nine-classes problem, the classification accuracy suffers from a deterioration of $4.08 \%$.

\section{DISCUSSION}

In literature, several researches have focused on different kinds of upper-limb prosthetic control based on the myoelectric signal. However, they have considered the most common levels of amputation, i.e. transradial and transhumeral levels. The present work, instead, has taken into account the shoulder disarticulation.

The primary aim of this study was to develop a SEMG classification system able to correctly estimate the intentional movement of a subject within a set of motion classes, at shoulder level. We considered, for data segmentation, a window length $\mathrm{L}=500 \mathrm{~ms}$ and an interval $\mathrm{I}=62 \mathrm{~ms}$. Feature extraction plays an important role in the pattern recognitionbased control system. Indeed, a large number of previous papers investigated which feature set returns the best classification performance, mainly for transradial and transhumeral amputation level. In this study, we have chosen a feature set among the ones proposed in the previous works that proved to produce the lower classification error. When applied to the shoulder disarticulation problem, our results showed that the feature set composed by SampEn, CC, RMS and WL, whose advantages are its temporal robustness, as demonstrated in [7], and its definition in time domain, which results into an easy implementation, provides good performance. In the four-classes problem all of the movements are correctly classified. The addition of the elevation to $90^{\circ}$ along the inclined plane, in the five-classes problem, yields an accuracy reduction equal to $2.61 \%$, due to the misclassification with the flexion to $90^{\circ}$ and the abduction to $90^{\circ}$. This result can be explained by the fact that this movement was a combination of the two classes just mentioned. Finally, in the nine-classes problem, the 
classification accuracy further decreases to $92.56 \%$. This value was mainly due to the misclassification of the flexion to $110^{\circ}$ with the flexion to $90^{\circ}$. This confusion can be ascribed to the closeness between these two motion classes.

Furthermore, when reducing the SEMG channels from eight to six, the results suggest that, even when choosing the signals with the less discriminating information, this reduction yielded a significant reduction in classification accuracy in the nine-classes problem; whereas, in the fourand the five-classes problem, it produced a very slight decline. Indeed, the more the classes of movement to be discriminated, the higher the number of acquisition channels required [2]. Therefore, in the four- and five-classes problems, only six of the eight channels can be used without compromising the classification accuracy. In this way, we can reduce the complexity, the weight, and the cost of the prosthesis [2].

\section{CONCLUSION}

This paper focused on the SEMG acquisition and signal processing aiming to implement a classification system based on pattern recognition to be used for controlling an active prosthesis for major upper limb amputations.

An EMG pattern recognition-based control system was implemented and evaluated in terms of classification accuracy.

Finally, the reduction of the number of acquisition SEMG channels and its influence on the classification performance was assessed. In a future work, the validity of the proposed method will be tested on shoulder amputees. Moreover, other applications of this movement intention classification approach will be explored for neuroprostheses development, i.e. for controlling Functional Electrical Stimulation based systems Error! Reference source not found., Error! Reference source not found., for controlling rehabilitation robotics or exoskeletons, as well as in the field of domotic systems, for assisting severely disabled persons.

\section{REFERENCES}

[1] K. Englehart and B. Hudgins, "A robust, real-time control scheme for multifunction myoelectric control," IEEE Trans. Biomed. Eng., vol. 50, no. 7, pp. 848-54, Jul. 2003.

[2] G. Li, "Electromyography pattern-recognition-based control of powered multifunctional upper-limb prostheses," Advances in Applied Electromyography, 2011, [Online] Available from: http://www.intechopen.com

[3]G. Gini, M. Arvetti, I. Somlai, and M. Folgheraiter, “Acquisition and analysis of EMG signals to recognize multiple hand movements for prosthetic applications," Applied Bionics and Biomechanics, no 9 , pp 145$155,2012$.

[4]B. Hudgins, P. Parker, and R. Scott, "A new strategy for multifunction myoelectric control," IEEE Trans. Biomed. Eng., vol. 40, no. 1, pp. 82-94, Jan. 1993.

[5]K. Englehart, B. Hudgins, and P.A. Parker, "A wavelet-based continuous classification scheme for multifunction myoelectric control," IEEE Trans. Biomed. Eng., vol. 48, no. 3, pp. 302-310, Mar. 2001.

[6]L. H. Smith, L. J. Hargrove, B. A. Lock, and T. A. Kuiken, "Determining the optimal window length for pattern recognition-based myoelectric control: balancing the competing effects of classification error and controller delay," IEEE Trans. Neural Syst. Rehabil. Eng., vol. 19, no. 2, pp. 186-192, Apr. 2011.

[7]A. Phinyomark , F. Quaine, S. Charbonnier, C. Serviere, F. TarpinBernard, and Y. Laurillau, "EMG feature evaluation for improving myoelectric pattern recognition robustness," Expert Systems with applications, vol. 40, no. 12, pp. 4832-4840, Sep. 2013.

[8]M. A. Oskoei and H. Hu, "Myoelectric control systems- a survey," Biomedical Signal Processing and Control (Elsevier), vol. 2, no. 4, pp. 275294, Sep. 2007.

[9]M. A. Oskoei and H. Hu, "Support vector machine-based classification scheme for myoelectric control applied to upper limb," IEEE Trans. Biomed. Eng., vol. 55, no. 8, pp. 1956-1965, Aug. 2008.

[10] P. Kauffmann, K. Englehart, and M. Platzer, "Fluctuating EMG signals: investigating long-term effects of pattern matching algorithms," in Proc.32nd Annu. Int. Conf. IEEE EMBS, Buenos Aires, Argentina, 2010, pp. 6357-6360.

[11] D. Peleg, E. Braiman, E. Yom-Tov, and G. F. Inbar, "Classification of finger activation for use in a robotic prosthesis arm," IEEE Trans. Neural Syst. Rehabil. Eng., vol. 10, no. 4, pp. 290-293, Dec. 2002.

[12] G.G. Yen and K.C. Lin, "Wavelet packet feature extraction for vibration monitoring," IEEE Trans. Ind. Electron., vol. 47, no. 3, pp. 650667, Jun. 2000.

[13] A. J. Jain, R. P. W. Duin, and J. Mao, "Statistical pattern recognition: A review," IEEE Trans. Pattern Anal. Mach. Intell., vol. 22, no. 1, pp. 4-37, Jan. 2000

[14] L. Hargrove, Y. Losier, B. A. Lock, K. Englehart, and B. Hudgins, "A real-time pattern recognition based myoelectric control usability study implemented in a virtual environment," in Proc. 29th Annual Int. Conf. IEEE EMBS, Lyon, France, 2007, pp. 4842-4845.

[15] T. A. Kuiken, G. Li, B. A. Lock, R. D. Lipschutz, L. A. Miller, K. A Stubblefield, K. B. Englehart, "Targeted muscle reinnervation for real-time myoelectric control of multifunction artificial arms," JAMA, vol. 301, no. 6, pp. 619-628, Feb. 2009.

[16] M. Barbero, R. Merletti, A. Rainoldi, Atlas of muscle innervation zones. Milan, Italy: Springer, pp. 103-120, 2012.

[17] J. T. Tou, R. C. Gonzalez, Pattern Recognition Principles, Reading: Addison-Wesley, 1974.

[18] M. Ferrarin, E. E. Pavan, R. Spadone, R. Cardini, C. Frigo, "Standing up exerciser based on Functional Electrical Stimulation and body weight relief," Med. Biol. Eng. Comput., vol.40, pp. 282-289, 2002.

[19] R. Riener, M. Ferrarin, M. Boll, E. Pavan, C. Frigo, "Patient-driven control of FES-supported standing up and sitting down: experimental results," IEEE Trans. Rehab. Eng., vol. 8, no. 4, pp. 523-529, 2000. 